Purpose We performed this study to investigate the potential predictors of long-term outcome in endovascular management of intracranial arteriovenous malformations (AVMs) with/without surgical resection in the pediatric population.

Materials and methods Consecutive pediatric patients $(<18$ years) presenting with intracranial AVMs verified by CT/MR/ DSA imaging studies were enrolled in the study. Baseline demographics, presenting symptoms, angioarchitecture characteristics [location, compact/diffuse nidus type, maximum nidus diameter, superficial/deep drainage, associated flow-induced aneurysm and/or venous varix/stenosis/ectasia, number of arterial feeders/draining veins, Spetzler-Martin Grading (SMG) scores, and ruptured/unruptured lesions], treatment strategy (endovascular embolization and/or surgical resection versus conservative management), procedural complication, recurrence, and long-term functional outcome were obtained. Ninety days modified Rankin Scale score of $\leq 2$ and achieving developmental milestones were used to determine good functional outcome as appropriate. Independent samples $\mathrm{T}$ test, chi square, and logistic regression analyzes were performed for statistical analyzes.

Results Fifty-eight cases (m/f: 38/20; mean age [(range) \pm SD: $10.99(0.5-17) \pm 4.09$ years] presented with 29 ruptured versus 29 unruptured AVMs. Presenting symptoms included severe headaches $(35.8 \%)$, occasional mild-moderate headache $(22.6 \%)$, incidental finding $(22.6 \%)$, seizure $(9.4 \%)$, focal neurological deficits (15.1\%), and loss of consciousness 20.8\%), respectively. Single or multi-session endovascular embolization was performed either alone or prior to surgical resection while three cases were managed conservatively. Procedural complication and lesion recurrence were encountered in $6.9 \%$ and $5.2 \%$ of cases, respectively. Good functional outcome was achieved in 49 (84\%) of cases. Angioarchitecture features of the lesions are presented in the table. No significant association was observed between SMG and final outcome $(\mathrm{P}=0.80)$. Regression analysis of clinical presentation, lesion characteristics, and treatment-related variables revealed that rupture and severe onset of headache were independent predictors of poor long-term functional outcome $(\mathrm{P}=0.006$, OR:3.5). Seven out of nine cases with poor clinical outcome

\begin{tabular}{|c|c|}
\hline Nidus Type $(n, \%)$ & Compact:52 (89.7\%) / Diffuse: 6 (10.3\%) \\
\hline Nidus Location $(n, \%)$ & Eloquent:33 (56.9\%) / Non-Eloquent: 25 (43.1\%) \\
\hline Lesion $\geq 3 \mathrm{~cm}(n, \%)$ & $22(33.9 \%)$ \\
\hline Drainage $(n, \%)$ & $\begin{array}{l}\text { Superficial: } 26 \text { (44.9\%) / Deep: } 15 \text { (25.8\%) / Superficial and } \\
\text { Deep : } 17 \text { (29.3\%) }\end{array}$ \\
\hline $\begin{array}{l}\text { Lesions with Multiple Arterial } \\
\text { Feeders }(n, \%)\end{array}$ & $43(74.1 \%)$ \\
\hline $\begin{array}{l}\text { Lesions with Multiple Draining Veins } \\
(\mathrm{n}, \%)\end{array}$ & $27(46.6 \%)$ \\
\hline Intranidal Aneurysm $(\mathrm{n}, \%)$ & $15(25.9 \%)$ \\
\hline Venous Stenosis $(n, \%)$ & $12(20.7 \%)$ \\
\hline Venous Varix $(n, \%)$ & $13(22.4 \%)$ \\
\hline Venous Ectasia $(\mathrm{n}, \%)$ & $20(34.5 \%)$ \\
\hline
\end{tabular}

Abstract 0-031 Figure 1 presented with ruptured AVMs with unfavorable preprocedural mRS score in eight of them.

Conclusions In our study, size, eloquent location, and complex angioarchitecture of intracranial AVMs were not predictors of outcome while presentation and ruptured/unruptured status of the lesions were significant predictors of long-term clinical outcome in treatment strategy with endovascular approach.

Disclosures A. Honarmand: None. S. Ansari: None. M. Hurley: None. P. Golnari: None. M. Potts: None. B. Jahromi: None. A. Shaibani: None.

\section{0-032 VENOUS BLOOD FLOW VISUALIZATION IN SIGMOID SINUS DIVERTICULUM USING MRI}

${ }^{1} \mathrm{M}$ Amans, ${ }^{1} \mathrm{E}$ Kao, ${ }^{1} \mathrm{~S}$ Kefayati, ${ }^{2} \mathrm{~K}$ Meisel, ${ }^{1} \mathrm{~F}$ Faraji, ${ }^{1} \mathrm{C}$ Glastonbury, ${ }^{1} \mathrm{M}$ Ballweber, ${ }^{1} \mathrm{~V}$ Halbach, ${ }^{1} \mathrm{D}$ Saloner. ${ }^{1}$ Radiology, UCSF, San Francisco, CA; ${ }^{2}$ Neurology, UCSF, San Francisco, CA

\subsection{6/neurintsurg-2016-012589.32}

Introduction/purpose Sigmoid sinus diverticulum (SSD) is one of the potentially treatable causes of pulsatile tinnitus (PT). How SSD causes PT is unknown, but it is thought to be secondary to aberrant blood flow in the diverticulum or parent sinus. We performed velocity field mapping using MR $4 \mathrm{D}$ Flow (MRV) and computational fluid dynamics (CFD) in cerebral venous sinuses and internal jugular veins (IJV). We aim to determine if a distinct blood flow pattern may be responsible for PT in SSD.

Materials and methods Patients suspected of venous etiology of PT underwent MRI at $3 \mathrm{~T}$, using contrast-enhanced MRA (timed to venous phase), MRV and CFD. SSD was confirmed on MRA. Flow pathlines were evaluated. In patients with confirmed SSD, additional CFD modelling was performed with the SSD excluded from the models.

Results Nineteen patients with suspected venous etiology of PT and 10 controls were evaluated. Six (31.5\%) had SSD and five of these had transverse sinus stenosis upstream from the SSD. These five patients also demonstrated a unique pattern of flow not seen in the controls characterized by:

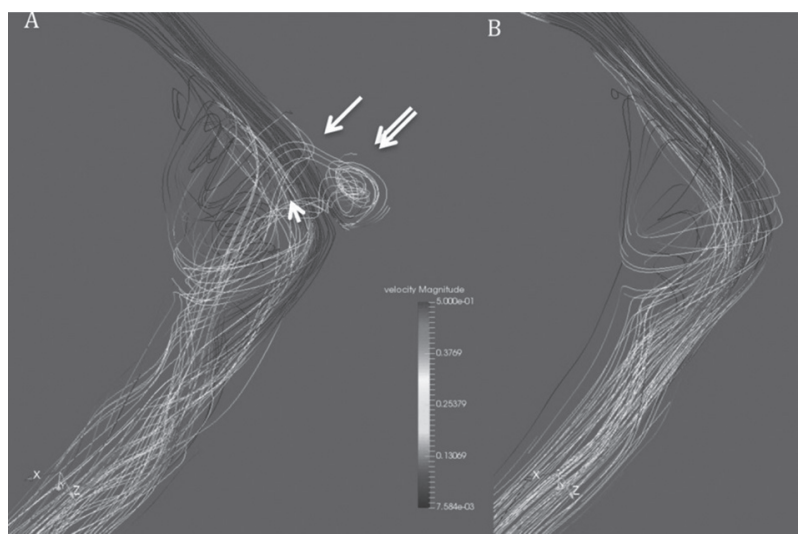

Abstract 0-032 Figure 1 Townes projection CFD analysis of a patient with a left SSD and an upstream stenosis in the transverse sinus. The pretreatment analysis (A) shows a jet of flow from the stenosis into the SSD (arrow), vortex of flow in the SSD (double arrow), and a vortex component of flow in the sigmoid sinus down-stream from the SSD (arrow head). The post-treatment model (B) shows absence of the flow in the SSD as well as decreased vortex component of flow in down-stream sigmoid sinus 
1. High velocity flow jet in an up-stream stenosis in the transverse sinus directed at the SSD opening,

2. Flow jet into the SSD along the long axis of the SSD, either anteriorly or laterally directed,

3. Vortex of flow in the SSD,

4. Prominent vortex component of flow in the sigmoid sinus downstream from SSD,

5. Vortex of flow in the jugular bulb

Three of the patients had simulated post-coil treatment models developed excluding the SSD from the models. CFD showed no flow in SSD and decreased vortex component of flow in the sigmoid sinus downstream from the SSD.

Conclusion PT caused by SSD may be caused by a unique flow pattern in the SSD and sinuses as visualized on both MRV and CFD.

Disclosures M. Amans: None. E. Kao: None. S. Kefayati: None. K. Meisel: None. F. Faraji: None. C. Glastonbury: None. M. Ballweber: None. V. Halbach: None. D. Saloner: None.

\section{0-033 JUGULAR VEIN FLOW PATTERNS IN PATIENTS WITH PULSATILE TINNITUS USING COMPUTATIONAL FLUID DYNAMICS}

${ }^{1} \mathrm{E}$ Kao, ${ }^{1} \mathrm{~S}$ Kefayati, ${ }^{2} \mathrm{~K}$ Meisel, ${ }^{1} \mathrm{M}$ Ballweber, ${ }^{1} \mathrm{~F}$ Faraji, ${ }^{1} \mathrm{~V}$ Halbach, ${ }^{1} \mathrm{D}$ Saloner, ${ }^{1} \mathrm{M}$ Amans. ${ }^{1}$ Radiology, UCSF, San Francisco, CA; ${ }^{2}$ Neurology, UCSF, San Francisco, CA

\subsection{6/neurintsurg-2016-012589.33}

Introduction/purpose Aberrations of venous anatomy can cause pulsatile tinnitus (PT). However, venous anatomy variation in patients without pulsatile tinnitus (PT) is extremely variable. Conventional imaging modalities, including cerebral angiography, provide little insight into the complex flow patterns in the cerebral veins. The aim of this study was to use subjectspecific contrast-enhanced MRA (CE-MRA) determined anatomy, and MRV-based inlet flow conditions to develop computational fluid dynamics (CFD) models of flow in subjects with suspected venous pulsatile tinnitus and subjects without pulsatile tinnitus to investigate how the geometry of the jugular vein affects flow.

Materials and methods 7 jugular veins (4 normal, 3 with pulsatile tinnitus) were imaged with CE-MRA. 2 D phase contrast MRV was also acquired transverse to the sigmoid sinus to determine inlet flow conditions. Surfaces were segmented using VMTK (Orobix, Bergamo, Italy) and Geomagic Design $\mathrm{X}$ (Geomagic, Rock Hill, USA). Tetrahedral meshing was also performed in VMTK, using a target edge-length of $0.6 \mathrm{~mm}$. CFD simulations were performed in FLUENT (ANSYS, Canonsburg, USA), using flow values obtained from the literature and in vivo measurements. Flow patterns in the jugular vein were characterized by their vortex core-lines, which were extracted from simulation data using Ensight (CEI, Apex, USA). Additional flow-parameters were calculated in MATLAB (Mathworks, Natick, USA) and pathlines visualized using Paraview (Kitware, New York, USA).

Results Patients with suspected venous PT had flow distinct patterns from those in normal subjects. Non-PT flow was characterized by organized redirection of flow from the sigmoid sinus along the curvature of the bulb into the jugular vein (A) with vortex cores in the jugular bulb (C). PT flow was characterized by larger helical flow structures throughout the proximal jugular vein created by flow directed perpendicular to the sigmoid sinus flow (B) and vortex cores that were more diffusely organized (D). High flow rates, and even turbulence, were noted in the proximal jugular vein near the level of the carotid bifurcation - where there was often pronounced narrowing of the jugular.

Conclusion The geometry of the jugular vein significantly affects the position, size, and length of the vortex cores. Our results suggest a link between geometry, flow, and PT.

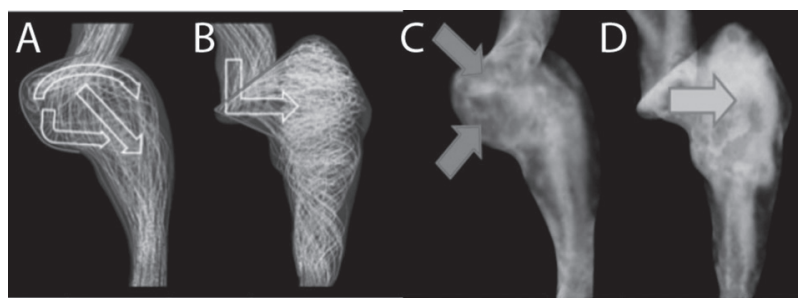

Abstract 0-033 Figure 1 Visualization of streamlines (A, B) and vortex cores by swirling strength $(C, D)$ in internal jugular veins of subjects without $(A, C)$ and with $(B, D)$ PT. White arrows $(A, B)$ reperent the general re-direction of flow from the sigmoid sinus into the jagular vein as dictated by the shape of the junction with the jugular bulb (red lines). Subjects without PT have strong vortex cores in jugular bulb (C, red arrows), while those with PT have larger, more diffuse vortex cores (D) that encompass nearly the entire proximal jugular vein (blue arrow).

Disclosures E. Kao: None. S. Kefayati: None. K. Meisel: None. M. Ballweber: None. F. Faraji: None. V. Halbach: None. D. Saloner: None. M. Amans: None.

\section{0-034 ABCIXIMAB THERAPY FOR THROMBOEMBOLIC COMPLICATIONS OF NEUROENDOVASCULAR PROCEDURES}

${ }^{1} \mathrm{~A}$ Kansagra, ${ }^{1} \mathrm{~T}$ Madaelil, ${ }^{1} \mathrm{D}$ Cross, III, ${ }^{1} \mathrm{C}$ Moran, ${ }^{2} \mathrm{C}$ Derdeyn. ${ }^{1}$ Washington University School of Medicine, Saint Louis, MO; ${ }^{2}$ University of lowa Hospitals and Clinics, Iowa City, IA

\subsection{6/neurintsurg-2016-012589.34}

Background Thromboembolic complications sustained during neuroendovascular procedures can result in postoperative infarcts if not promptly recognized and treated. Treatment commonly involves glycoprotein IIb/IIIa inhibitors such as abciximab. We aimed to retrospectively review angiographic and clinical outcomes following abciximab administration for thromboembolic complications at our institution.

Methods Neuroendovascular cases with thromboembolic complications treated with abciximab over a 132 month period were identified using a search of a comprehensive, prospectively maintained case $\log$ and all angiography records for the terms "abciximab" or "ReoPro." Intraoperative intra-arterial (IA) administration typically involved slow infusion of $10 \mathrm{mg}$ abciximab over 5 to 10 minutes. Intraoperative intravenous (IV) administration $0.25 \mathrm{mg} / \mathrm{kg}$ abciximab. Postoperative IV infusion typically involved $10 \mathrm{mcg} / \mathrm{min}$ infusion of abciximab. All relevant clinical notes and neuroimaging were reviewed.

Results Of 19,566 neuroendovascular procedures performed during the review period, $48(0.25 \%)$ involved abciximab administration for thromboembolic complications. 65\% (31/48) involved IA administration, 21\% (10/48) were IV only, and $15 \%$ (7/48) were combined IA and IV. Intraoperative treatment was supplemented with postoperative abciximab infusion in $13 \%(6 / 48)$ patients. Angiographic improvement was seen in $92 \%(44 / 48)$ cases, including 65\% (31/48) with complete 